\title{
Análisis cinético y estadístico de la precipitación en una aleación de Cu-0,49Co-0,44Ti, mediante microcalorimetría y medidas de microdureza ${ }^{(\bullet)}$
}

\author{
E. Donoso* y G. Díaz*
}

\begin{abstract}
Resumen
Mediante calorimetría diferencial de barrido (DSC) se estudió la cinética de precipitación de átomos de cobalto y titanio a partir de una solución sólida de $\mathrm{Cu}-0,49 \mathrm{Co}-0,44 \mathrm{Ti}$, templada desde $1.173 \mathrm{~K}$. El análisis de las curvas calorimétricas muestran la presencia de una reacción exotérmica la cual se atribuye a la formación de partículas de CoTi en la matriz de cobre. La energía de activación de la reacción fue estimada a partir del método de Kissinger modificado. Los parámetros cinéticos fueron calculados mediante el formalismo de Johnson- Mehl-Avrami. Por otra parte, se efectuó un análisis estadístico del proceso de precipitación, mediante medidas de microdureza Vickers, empleando una función de distribución de probabilidad de Weibull. Se estimaron los parámetros de Weibull mediante el método de mínimos cuadrados. La bondad de ajuste se analizó considerando el test de chi-cuadrado con un nivel de confianza del $95 \%$. A medida que se incrementó el tiempo de envejecimiento, para igual temperatura de recocido, incrementó el valor del módulo de Weibull, lo cual puede atribuirse a la precipitación de la fase CoTi.
\end{abstract}

Palabras clave $\quad$ Cobre; $\mathrm{Cu}$-Co-Ti; Precipitación; Microdureza; Estadística de Weibull.

\section{Kinetics and statistical analysis of precipitation in a Cu-0.49Co-0.44Ti alloy, by microcalorimetry and microhardness measurements}

\begin{abstract}
Starting with a solid solution of $\mathrm{Cu}-0,49 \mathrm{Co}-0,44 \mathrm{Ti}$ tempered from $1173 \mathrm{~K}$, the kinetics of precipitation of atoms of cobalt and titanium was studied by means of differential scanning calorimetry (DSC). The analysis of the calorimetric curves show the presence of an exothermal reaction that is attributed to the formation of particles of CoTi in the copper matrix. The energy of activation of the reaction was estimated by means of a modified method of Kissinger. The kinetic parameters were estimated with the use of the formalism of Johnson-Mehl-Avrami. On the other hand, a statistical analysis of the process of precipitation was performed by measuring the microhardness Vickers, employing a Weibull probability distribution function. Using minimum square method the Weibull parameters were estimated. The goodness of fit was analyzed by using the Chi square test with a condidence level of 95 percent. Increasing the aging time, for the sam annealing temperature, the Weibull modulus increase too, which may be attributed to precipitation of CoTi phase.
\end{abstract}

Keywords Copper; Cu-Co-Ti; Precipitation; Microhardness; Weibull statistics.

\section{INTRODUCCIÓN}

El desarrollo de aleaciones ternarias de alta resistencia está siendo motivo de variados estudios desde mediados de la década pasada. La mayor parte de ellas fundamenta su resistencia en la formación de precipitados binarios y/o ternarios de extrema fineza, resistentes a ser cortados por las dislocaciones, confiriéndole al material un elevado límite de fluencia. Un buen ejemplo de este efecto son las aleaciones de $\mathrm{Cu}$ $\mathrm{Co}-\mathrm{Si}^{[1-3]}, \mathrm{Cu}-\mathrm{Co}-\mathrm{Ti}^{[4-7]}, \mathrm{Cu}-\mathrm{Al}-\mathrm{Co}^{[8]}, \mathrm{Cu}-\mathrm{Ni}-\mathrm{Al}^{[9-11]}$, etc. En el caso de las aleaciones $\mathrm{Cu}-\mathrm{Co}-\mathrm{Ti}$, el proceso de precipitación ha sido estudiado por difracción de rayos $\mathrm{X}^{[12-14]}$, microscopía electrónica de transmisión (TEM) y energía dispersiva de rayos $\mathrm{X}(\mathrm{EDX})^{[4-6]}$. Estos estudios indican que, dependiendo de las concentraciones de cobalto y titanio se podrían formar precipitados esféricos con estructura cúbica CoTi y/o $\mathrm{Co}_{2} \mathrm{Ti}$.

(•) Trabajo recibido el día 21 de mayo de 2009 y aceptado en su forma final el día 22 de julio de 2009.

* Universidad de Chile, Facultad de Ciencias Físicas y Matemáticas, Departamento de Ciencia de los Materiales, Av. Tupper 2069, casilla 2777, Santiago-Chile, e-mail: edonoso@ing.uchile.cl. 
Por otra parte, los resultados experimentales de Mineau et al. ${ }^{[4]}$ sugieren que el límite de solubilidad de la fase CoTi en cobre, en el rango de temperatura de 773 a $1.230 \mathrm{~K}$, es mucho más baja que las solubilidades individuales del titanio y del cobalto en la matriz de cobre, con una fuerte tendencia a formar CoTi en la aleación ternaria $\mathrm{Cu}$-Co-Ti en vez de partículas de $\mathrm{Co}, \mathrm{Cu}_{3} \mathrm{Ti}_{\text {o }} \mathrm{Cu}_{4} \mathrm{Ti}$.

En general, prácticamente, cualquier ensayo mecánico está sujeto a dispersiones y fluctuaciones estadísticas. Estas deben tratarse mediante los formalismos apropiados, de modo tal que se pueda dar respuesta satisfactoria frente a la caracterización de algún material, al hacer empleo de alguna propiedad característica de éste. El primero en hacerse cargo de este problema fue Weibul[ ${ }^{[15]}$, al estudiar las elevadas dispersiones que tenían las tensiones de fractura de los materiales frágiles, proponiendo una función de distribución de probabilidad que lleva actualmente su nombre. Este trabajo pionero se extendió rápidamente a materiales con diverso comportamiento a la fractura. Hoy, incluso con los sofisticados refinamientos en la determinación de las tensiones características de los materiales, se emplea la función de distribución de probabilidad acumulativa de Weibull en la caracterización de nanomateriales ${ }^{[16]}$. No obstante lo anterior, algunos trabajos señalan la insuficiencia de soporte experimental de la función de Weibull y la no existencia de justificación clara para su elección frente, por ejemplo, a la función de distribución normal ${ }^{[17]}$. En un reciente trabajo ${ }^{[18]}$ se discute, precisamente, respecto de las ventajas de una función de Weibull frente a una función normal, para el caso del factor de intensidad crítico de tensiones en modo I.

La microdureza Vickers también ha sido estudiada desde un punto de vista probabilístico y se han empleado como funciones de distribución de probabilidad la normal, la log normal y la de Weibull. Schneider et al.$^{[19]}$ estudiaron la función de distribución de ambas diagonales, que deja el indentador Vickers en un material estándar de dureza y, a partir de ella, infirieron que la distribución que mejor se ajustaba a los datos de microdureza Vickers era una función de distribución normal. Lin et al. ${ }^{[20]}$ y Tóth et al. ${ }^{[21]}$ también analizaron la microdureza Vickers, conjuntamente con la función de Weibull.

El principal objetivo de este trabajo es el estudio de la cinética y análisis estadístico del proceso de precipitación del CoTi, a partir de soluciones supersaturadas de $\mathrm{Cu}-\mathrm{Co}-\mathrm{Ti}$, con composición perteneciente al sistema cuasi binario $\mathrm{Cu}-\mathrm{CoTi}$, utilizando medidas de microdureza Vickers y curvas calorimétricas.

\section{PROCEDIMIENTO EXPERIMENTAL}

La aleación utilizada se preparó en un horno de inducción, en atmósfera inerte (Ar), a partir de cobre electrolítico (99,95 \% de pureza), una aleación previa de $\mathrm{Cu}-10 \%$ wt. cobalto y titanio de alta pureza. El lingote se recoció a $1.223 \mathrm{~K}$ durante $24 \mathrm{~h}$ a fin de homogeneizarlo y se enfrió en el horno hasta la temperatura ambiente. Después de un análisis químico se encontró que la aleación contenía $\mathrm{Cu}-0,49 \%$ at. Co$0,44 \%$ at. $\mathrm{Ti}(\mathrm{Cu}-0,49 \mathrm{Co}-0,44 \mathrm{Ti})$. Posteriormente, el material se laminó en frío hasta $3 \mathrm{~mm}$ de espesor, con recocidos intermedios de $1 \mathrm{~h}$ a $1.173 \mathrm{~K}$. Después del último recocido, el material se templó en agua. El análisis microcalorimétrico de las muestras se realizó en un analizador térmico Dupont 2000, con velocidades de calentamiento lineal $(\beta)$ de 0,$08 ; 0,17 ; 0,33 ; 0,50$ y $0,67 \mathrm{~K} \mathrm{~s}^{-1}$, desde temperatura ambiente hasta $900 \mathrm{~K}$. Se usaron discos de $6 \mathrm{~mm}$ de diámetro y $3 \mathrm{~mm}$ de espesor. A fin de aumentar la precisión de las medidas, se utilizó como referencia un disco de cobre de alta pureza recocido durante un largo período. Para minimizar la oxidación de las muestras, se hizo pasar argón por el calorímetro $\left(10^{-4} \mathrm{~m}^{3} \mathrm{~min}^{-1}\right)$. Las curvas calorimétricas resultantes fueron convertidas en curvas de capacidad calórica diferencial vs. temperatura. La capacidad calórica remanente, es decir, la capacidad calórica diferencial $\Delta \mathrm{C}_{\mathrm{p}}$, representa el calor asociado con las reacciones en estado sólido que ocurren durante el experimento DSC. Las curvas calorimétricas presentadas en este trabajo son curvas corregidas por medio del método sugerido en la literatura ${ }^{1}$ y 22$]$.

Las medidas de microdureza fueron realizadas, a temperatura ambiente, en un microdurómetro Duramin $-1 /-2$ Struers de alta precisión, empleando una carga de 1,96 N durante $10 \mathrm{~s}$ sobre muestras planas en forma de discos. Las medidas fueron realizadas en el material templado y en muestras recocidas durante diferentes tiempos. Las temperaturas de recocido, sobre muestras previamente templadas desde $1.173 \mathrm{~K}$, fueron 653,723 y $793 \mathrm{~K}$, arrojando un total de seis muestras de la misma aleación sometidas a diversos periodos de envejecimiento. Se efectuaron 20 mediciones de microdureza a cada muestra de la aleación a fin de proceder a su análisis estadístico.

\section{RESULTADOS Y DISCUSION}

\subsection{Análisis calorimétrico}

La figura 1 muestra termogramas típicos para la aleación templada, en forma de capacidad calórica diferencial $\left(\Delta \mathrm{C}_{\mathrm{p}}\right)$ vs. temperatura, para diferentes 


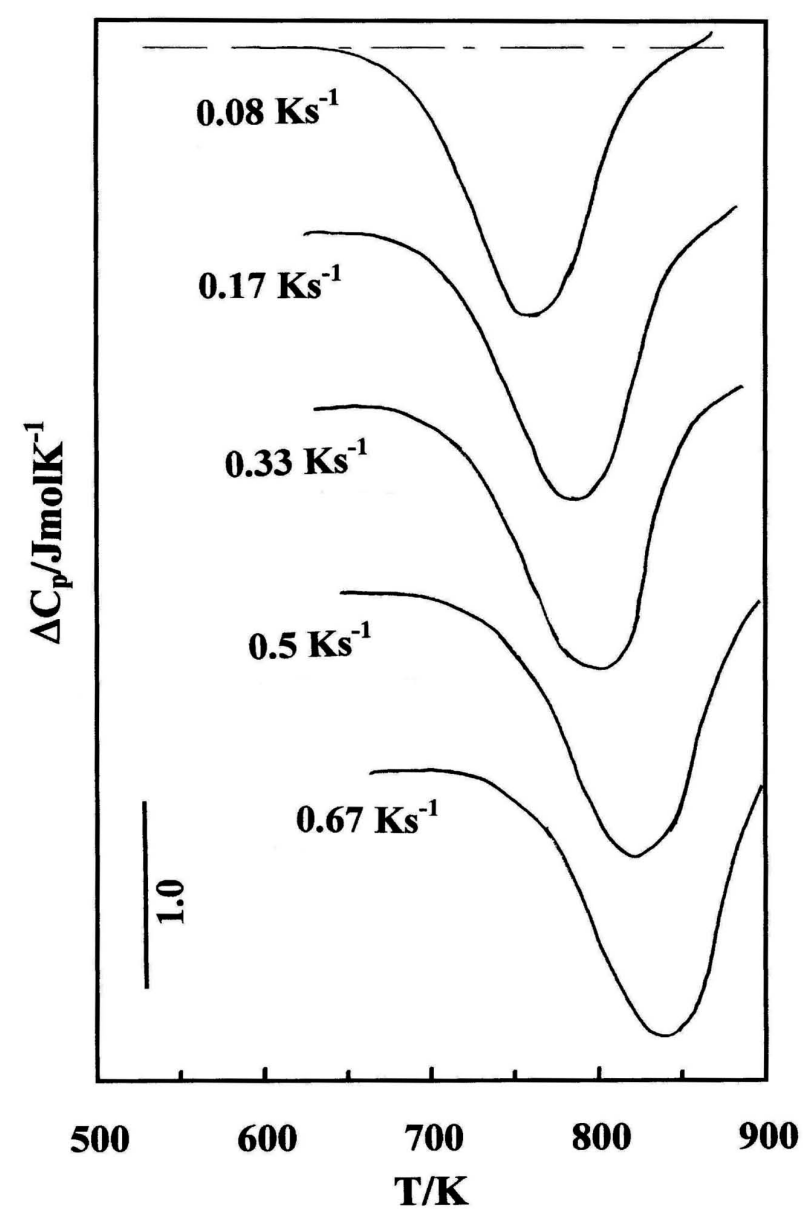

Figura 1. Curvas calorimétricas a diferentes velocidades de calentamiento obtenidas para aleaciones de $\mathrm{Cu}-1 \% \mathrm{CoTi}$, previamente templadas desde $1.173 \mathrm{~K}$.

Figure 1. Calorimetric curves at different heating rates obtained for $\mathrm{Cu}-1 \%$ CoTi samples previously quenched from $1,173 \mathrm{~K}$.

velocidades de calentamiento lineal $(\beta)$. Las curvas DSC muestran, en el rango de temperaturas barridas, un efecto exotérmico (etapa 1), que de acuerdo a los antecedentes descritos en la literatura ${ }^{[4-7]}$ podría atribuirse a la precipitación de partículas de
CoTi. Se puede observar que el calor asociado a cada una de las etapas es independiente de la velocidad de calentamiento. Por otra parte, el hecho de que las temperaturas de los picos de DSC se desplacen a temperaturas más altas al aumentar la velocidad de calentamiento pone de manifiesto el control cinético del proceso ${ }^{[23]}$.

La absorción de entalpía asociada a las diferentes etapas se evaluó considerando el área bajo la curva $\Delta \mathrm{C}_{\mathrm{p}}$ vs. T entre la temperatura a la cual la curva cruza la línea base y la temperatura a la cual la energía es absorbida a una tasa constante desde la línea base. Los valores de las entalpías de reacción, $\Delta \mathrm{H}$, se muestran en la tabla I. Dichos valores mostrados para cada velocidad de calentamiento, $b$, representan el valor medio de 5 experiencias calorimétricas.

El análisis cinético de las curvas DSC mostradas en la figura 1 , se ha realizado tomando en consideración que la velocidad de reacción es $\mathrm{d} \alpha / \mathrm{dt}=$ $(1 / S)\left(d_{t} / d t\right)$, donde a es la cantidad que ha reaccionado al tiempo $t$, da $\mathrm{d}_{\mathrm{t}} / \mathrm{dt}$ es la velocidad del flujo calórico, $a_{t}$ es el área bajo la curva al tiempo t, y $S$ es el área total bajo la curva. Integrando la ecuación anterior se tiene $\alpha=a_{t} / S$.

El valor de la energía de activación $\mathrm{E}_{\mathrm{a}}$, que se requiere para realizar el análisis cinético, puede ser evaluado a partir del método de Kissinger modificado por Mittenmeijer et al ${ }^{[24]}$ :

$$
\ln \left(\frac{\mathrm{T}_{\mathrm{p}}^{2}}{\beta}\right)=\frac{\mathrm{E}_{\mathrm{a}}}{\mathrm{RT}}+\ln \left(\frac{\mathrm{E}}{\mathrm{RA}}\right)
$$

Siendo $T_{p}$ la temperatura a la cual la velocidad de reacción es máxima, $\beta=\mathrm{dT} / \mathrm{dt}$, A es el factor pre-exponencial de Arrhenius, y $\mathrm{R}$ la constante de los gases. Por lo tanto, $\mathrm{E}_{\mathrm{a}}$ y $\mathrm{A}$ pueden ser evaluados a partir de la curva $\ln \left(\mathrm{T}_{\mathrm{p}}{ }^{2} / \beta\right)$ vs. $1 / \mathrm{T}_{\mathrm{p}}$, cuya pendiente es igual a $\mathrm{E}_{\mathrm{a}} / \mathrm{R}$. Dicha curva se muestra en la figura 2, obteniéndose una energía de activación $\mathrm{E}_{\mathrm{a}}=130,6 \mathrm{~kJ} \mathrm{~mol}^{-1}$ y el factor pre-exponencial $A=2,5 \times 10^{6} \mathrm{~s}^{-1}$. Se puede observar que la energía de activación evaluada por el método de Kissinger resultó más baja que las energías de di-

Tabla I. Entalpías de reacción obtenidas a partir de las curvas DSC

Table I. Reaction enthalpies obtained from DSC curves

\begin{tabular}{lccccc}
\hline $\mathbf{b}\left(\mathrm{Ks}^{-1}\right)$ & 0.08 & 0.17 & 0.33 & 0.50 & 0.67 \\
\hline$\Delta \mathbf{H}\left(\mathrm{Jmol}^{-1}\right)$ & $133 \pm 7$ & $128 \pm 6$ & $127 \pm 6$ & $132 \pm 7$ & $129 \pm 7$ \\
\hline
\end{tabular}

* Los valores representan el promedio de 5 experiencias calorimétricas para cada b. 


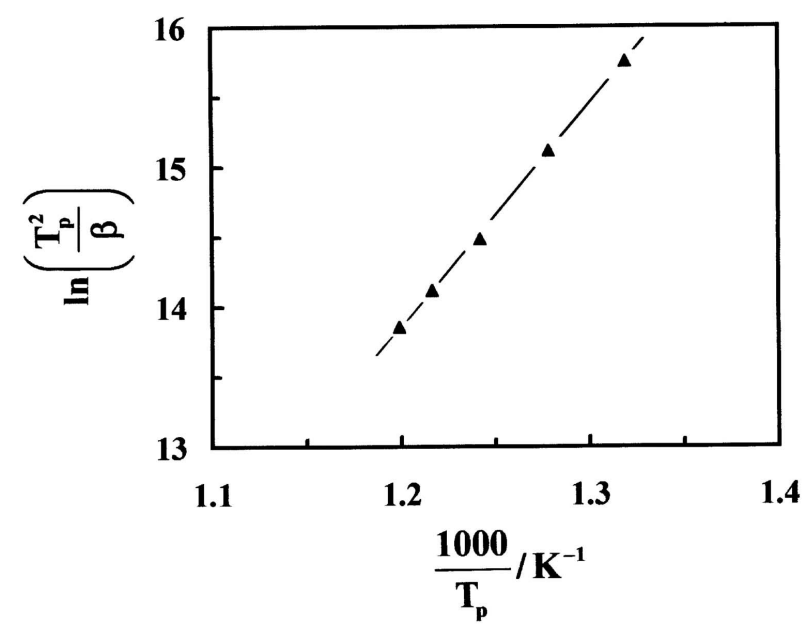

Figura 2. Gráfico de Kissinger modificado utilizado para evaluar la energía de activación y el factor pre-exponencial.

Figure 2. Modified Kissinger plot for evaluating activation energy and pre-exponential factor.

fusión de Co en $\mathrm{Cu}\left(\mathrm{E}_{\mathrm{Co} \rightarrow \mathrm{Cu}}=200,6 \mathrm{~kJ} \mathrm{~mol}^{-1}\right)$ y Ti en $\mathrm{Cu}\left(\mathrm{E}_{\mathrm{T} \rightarrow \mathrm{Cu}}=200,3 \mathrm{~kJ} \mathrm{~mol}^{-1}\right)$, ambas estimadas a partir de las correlaciones de Brown y Ashby ${ }^{[25]}$. Este hecho puede ser atribuido a la fuerte contribución de las vacantes introducidas por temple.

El análisis cinético se realizó empleando la ecuación usual de Johnson-Mehl-Avrami (JMA), utilizada para reacciones heterogéneas, bajo condiciones no isotermales ${ }^{[7]}$ :

$$
\ln [-\ln (1-\alpha)]=n \ln A+n \ln \left[\frac{R T^{2}}{E_{a} \beta} \exp \left(-\frac{E_{a}}{R T}\right)\right]
$$

Donde $\alpha$ es la fracción reaccionada, $\mathrm{n}$ es una constante que depende del modelo de reacción y $\mathrm{A}$ es el factor pre-exponencial de Arrhenius. El término $\theta=$ $\left.T^{2} R / \beta E_{a} \exp \left[-E_{a} / R T\right]\right)$ representa el tiempo reducido introducido por Ozawa ${ }^{[26]}$. Gráficos de $\ln \ln [1 /(1-\alpha)]$ vs. $\ln \theta$, después de haber introducido el valor de $\mathrm{E}_{\mathrm{a}}$ obtenido por el método de Kissinger, da una recta de pendiente $\mathrm{n}$ e intersección $\mathrm{n} \ln \mathrm{A}$, como se muestra en la figura 3. El valor de $n$ y $A$ evaluados bajo este procedimiento resultaron $\mathrm{n}=1,16$ y $\mathrm{A}=3,4 \times 10^{6} \mathrm{~s}^{-1}$. El valor de $\mathrm{n}$, cercano a 1,0 es indicativo de un proceso de nucleación y crecimiento a partir de una solución sólida ${ }^{[27]}$.

\subsection{Microdureza Vickers, análisis estadístico}

Adicionalmente, se realizaron medidas isotérmicas de microdureza Vickers después de efectuar en el

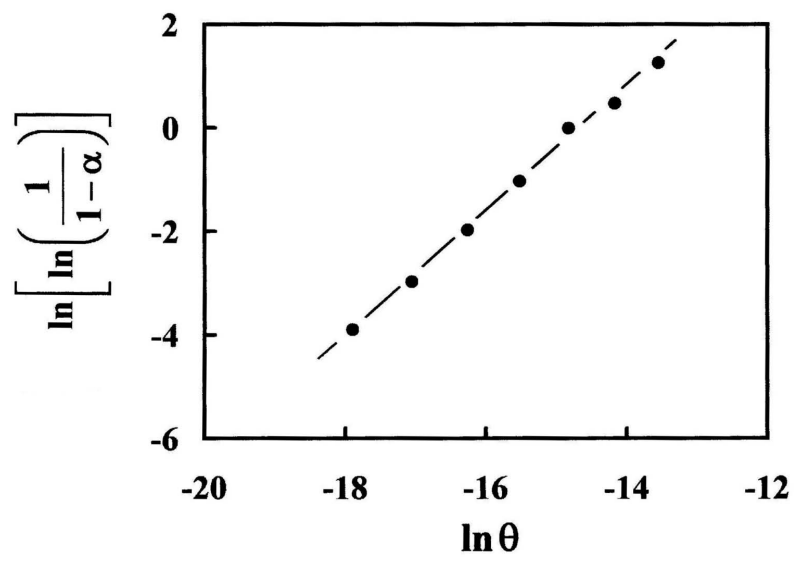

Figura 3. Curva de $\ln [\ln (1 / 1-a)]$ calculada a partir de las curvas DSC en función de $\ln \theta$.

Figure 3. Plot of $\operatorname{In}[\operatorname{In}(1 / 1-a)]$ calculated from DSC curves as a function of $\ln \theta$.

material templado tratamientos de envejecimientos a diferentes temperaturas. Los valores medios de dichas medidas, $\overline{\mathrm{H}}$, se muestran en la tabla II. Las temperaturas de envejecimiento a 723 y $793 \mathrm{~K}$ caen dentro del rango de las temperaturas de la reacción de las curvas calorimétricas mostradas en la figura 1 . La temperatura de envejecimiento a $653 \mathrm{~K}$ por 0 ks correspondería al material con solución sólida $\alpha$, donde los átomos de cobalto y titanio se encuentran solubles en la matriz de cobre; después de 3,6 ks de recocido habría una fuerte tendencia a formar partículas de CoTi, resultando un incremento de dureza de 101 a $121 \mathrm{HV}$. Por otra parte, se puede observar un incremento de dureza máximo, de 101 a 205 HV, después de recocer el material a $723 \mathrm{~K}$ durante $12,6 \mathrm{ks}$, lo cual puede atribuirse a la formación de partículas de CoTi.

Las medidas de microdureza Vickers pueden ser analizadas mediante el empleo de funciones de distribución de probabilidad, específicamente cuando las mediciones están afectadas a dispersiones considerables y éstas no tienen vinculación con el proceso de medida sino con la constitución propia del material.

La función de distribución de probabilidad acumulativa de Weibull tiene la siguiente expresión:

$$
F(H)=1-\exp \left\{-\left(\frac{H}{H_{0}}\right)^{m}\right\}
$$

Donde, F es la probabilidad, $\mathrm{H}$ es la variable aleatoria, en este caso corresponde a la microdureza Vickers, $\mathrm{H}_{0}$ y m son los parámetros de Weibull, $\mathrm{H}_{0}$ es el parámetro de escala y m es el módulo de Weibull. En general, 
ANÁLISIS CINÉTICO Y ESTADÍSTICO DE LA PRECIPITACIÓN EN UNA ALEACIÓN DE CU-0,49C0-0,44TI, MEDIANTE MICROCALORIMETRÍA Y MEDIDAS DE MICRODUREZA KINETICS AND STATISTICAL ANALYSIS OF PRECIPITATION IN A CU-0.49CO-0.44TI ALLOY, BY MICROCALORIMETRY AND MICROHARDNESS MEASUREMENTS

Tabla II. Valores medios de la microdureza Vickers $\overline{\mathrm{H}} \vee$ y su desviación estándar $\Delta \overline{\mathrm{H}} \vee$, parámetros de Weibull, $\mathrm{m} \mathrm{y} \mathrm{H}_{0}$ y sus dispersiones $\Delta \mathrm{m}$ y $\Delta \mathrm{H}_{0}$, para diversas temperaturas y tiempos de envejecimiento. Se indica, además, el resultado de aplicar el test de hipótesis de bondad de ajuste de chi-cuadrado con un $95 \%$ de nivel de confianza

Table II. Mean values of Vickers's microhardness $\overline{\mathrm{H}} \mathrm{v}$ and its standard deviation $\Delta \overline{\mathrm{H}} \mathrm{v}$, Weibull's parameters $\mathrm{m}$ and $\mathrm{H}_{0}$ and its dispersions $\Delta \mathrm{m}$ and $\Delta \mathrm{H}_{0}$ for different temperatures and aging times. The chi-square goodness of fit test with $95 \%$ of confidence level is given

\begin{tabular}{|c|c|c|c|c|c|c|c|c|}
\hline \multirow[t]{2}{*}{$\begin{array}{l}\mathrm{T} \\
\mathrm{K}\end{array}$} & \multirow[t]{2}{*}{$\begin{array}{c}t \\
k s\end{array}$} & \multicolumn{2}{|c|}{ Microdureza Vickers } & \multicolumn{4}{|c|}{$\begin{array}{l}\text { Parámetros de Weibull } \\
\text { y sus dispersiones }\end{array}$} & \multirow{2}{*}{$\begin{array}{c}\begin{array}{c}\text { Test de } \\
\text { hipótesis }\end{array} \\
\chi_{95 \%}^{2}\end{array}$} \\
\hline & & $\overline{\mathrm{H}} \mathrm{v}$ & $\Delta \overline{\mathrm{H}} \mathrm{v}$ & $\mathrm{m}$ & $\mathrm{H}_{0}$ & $\Delta \mathrm{m}$ & $\Delta \mathrm{H}_{0}$ & \\
\hline 653 & $\begin{array}{l}0,0 \\
3,6\end{array}$ & $\begin{array}{l}101 \\
121\end{array}$ & $\begin{array}{c}17,7 \\
8,0\end{array}$ & $\begin{array}{r}6,8 \\
17,9\end{array}$ & $\begin{array}{l}108 \\
124\end{array}$ & $\begin{array}{l}2,1 \\
3,4\end{array}$ & $\begin{array}{l}4,2 \\
3,7\end{array}$ & $\begin{array}{c}\text { Rechazado } \\
\text { Aceptado }\end{array}$ \\
\hline 723 & $\begin{array}{r}0,6 \\
12,6\end{array}$ & $\begin{array}{l}114 \\
205\end{array}$ & $\begin{array}{l}15,9 \\
17,0\end{array}$ & $\begin{array}{r}8,3 \\
13,8\end{array}$ & $\begin{array}{l}123 \\
213\end{array}$ & $\begin{array}{l}1,3 \\
2,4\end{array}$ & $\begin{array}{l}2,6 \\
4,5\end{array}$ & $\begin{array}{l}\text { Aceptado } \\
\text { Aceptado }\end{array}$ \\
\hline 793 & $\begin{array}{l}0,6 \\
1,5\end{array}$ & $\begin{array}{l}118 \\
133\end{array}$ & $\begin{array}{l}6,1 \\
6,4\end{array}$ & $\begin{array}{l}23,3 \\
24,1\end{array}$ & $\begin{array}{l}120 \\
135\end{array}$ & $\begin{array}{l}1,9 \\
1,7\end{array}$ & $\begin{array}{l}3,1 \\
3,9\end{array}$ & $\begin{array}{l}\text { Aceptado } \\
\text { Aceptado }\end{array}$ \\
\hline
\end{tabular}

* Representa el promedio de 20 medidas de microdureza.

los parámetros de Weibull tienen que ver con el proceso de fabricación del material, en este caso, con los tratamientos térmicos a que han sido sometidas las diversas muestras de la aleación en estudio.

Varios son los métodos que se han empleado en la determinación de los parámetros de Weibull: Uno de los más empleados, en razón de su simplicidad, es el de regresión lineal mediante mínimos cuadrados. Para realizar lo anterior se linealiza la ecuación (3) de la siguiente manera:

$$
\ln \left[\ln \left(\frac{1}{1-\mathrm{F}(\mathrm{H})}\right)\right]=\mathrm{m} \operatorname{lnH}-\mathrm{mlnH}_{0}
$$

Así, se obtienen el parámetro m como la pendiente de la recta de regresión mínimo cuadrática y el parámetro $\mathrm{H}_{0}$ a partir del punto de intersección de dicha recta.

La introducción de los datos experimentales de microdureza se hace de la siguiente manera: para cada conjunto de las 20 indentaciones realizadas a cada una de las muestras se procede a ordenarlas de menor a mayor valor de microdureza Vickers. Como no se conocen las verdaderas probabilidades asociadas a cada valor de microdureza hay que emplear un estimador para ellas. Se sabe, de estudios previos, que uno de los estimadores de menor sesgo, en muestras con un número mínimo de 20 datos, es el siguiente:

$$
F=\frac{p-0,5}{N}
$$

donde, $\mathrm{p}$ es el número de orden en el conjunto de datos experimentales agrupados ascendentemente y $\mathrm{N}$, en este caso igual a 20 , es el número de ensayos, aquí, número de microdurezas Vickers practicadas sobre cada muestra de aleación considerada.

La etapa siguiente es la confección de los diagramas de Weibull, para ello se emplean las ecuaciones (4) y (5), y los respectivos datos de microdureza Vickers. El diagrama de Weibull se consigue graficando $\ln \ln [1 /(1-\mathrm{F}(\mathrm{H}))]$ vs. $\ln \mathrm{H}$. En la figura 4 se muestra el diagrama de Weibull de la microdureza Vickers de la aleación bajo estudio, previamente templada desde $1.173 \mathrm{~K}$ con un recocido posterior a 723 K y un tiempo de envejecimiento de 12,6 ks. Similares diagramas se obtuvieron para otros cinco tratamientos térmicos, como se indica en el procedimiento experimental. Desde un punto de vista práctico, frente a consideraciones, por ejemplo, de diseño, es interesante mostrar, directamente en el diagrama de Weibull, la probabilidad acumulativa $\mathrm{F}(\mathrm{HV})$ en función de la variable aleatoria, en este caso, microdureza Vickers HV.

Por otra parte, como se puede observar en la tabla III, los valores de microdureza Vickers, para las temperaturas de recocido de 723 y $793 \mathrm{~K}$, se incrementan con el tiempo hasta alcanzar un valor máxi- 


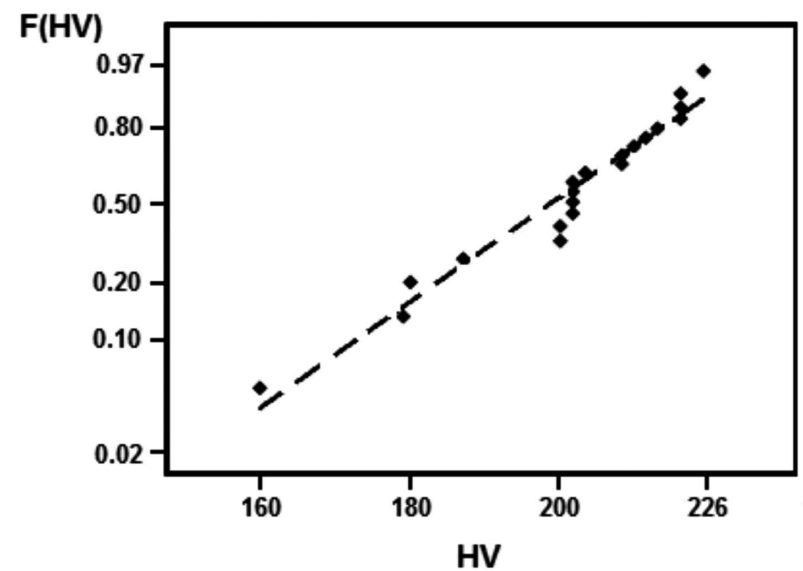

Figura 4. Diagrama de probabilidad acumulativa de Weibull, $F(H V)$ de la microdureza Vickers, HV, para muestras previamente templadas desde $1.173 \mathrm{~K}$ y envejecidas a $723 \mathrm{~K}$ durante $12,6 \mathrm{ks}$.

Figure 4. Weibull's cumulative probability diagram, $\mathrm{F}(\mathrm{HV})$ of Vickers microhardness, for samples previously quenched from $1,173 \mathrm{~K}$ and aged at $723 \mathrm{~K}$ for $12.6 \mathrm{ks}$.

Tabla III. Valores de microdureza Vickers obtenidos a diferentes temperaturas y tiempos de envejecimiento en muestras previamente templadas desde $1.173 \mathrm{~K}$

Table III. Vickers microhardness values obtained at different temperatures and aging times for samples previously quenched from $1,173 \mathrm{~K}$

\begin{tabular}{ccc}
\hline & \multicolumn{2}{c}{ Microdureza Vickers (HV) } \\
\cline { 2 - 3 } $\mathbf{t}(\mathbf{k s})$ & $\mathbf{7 2 3 \mathbf { K }}$ & $\mathbf{7 9 3} \mathbf{~ K}$ \\
\hline 0 & 101 & 101 \\
0,6 & 114 & 118 \\
1,5 & 118 & 133 \\
2,4 & 126 & 146 \\
5,4 & 158 & 190 \\
12,6 & 205 & 153 \\
15,0 & 198 & 147 \\
16,8 & 184 & - \\
\hline
\end{tabular}

* Los valores de HV representan el promedio de 20 medidas de microdureza.

mo. Dichos valores son indicativos de que, en cada caso, se ha alcanzado un grado de equilibrio de la precipitación. El máximo de endurecimiento se produjo con un recocido a $723 \mathrm{~K}$ durante $12,6 \mathrm{ks}$.
Junto con estimar los parámetros de Weibull hay que estimar sus dispersiones puesto que ellos no están exentos de presentar fluctuaciones. La estimación de la dispersión de los parámetros se consigue empleando la matriz de información de Fischer. El procedimiento es como sigue. Los elementos de la matriz de Fischer se obtienen mediante la expresión:

$$
r_{i j}=-N E\left[\frac{\partial^{2} \ln \frac{d F}{d H}\left(H ; m, H_{0}\right)}{\partial \theta_{i} \partial \theta_{j}}\right] ;\{\theta\}=\left\{m, H_{0}\right\}
$$

donde, $\mathrm{N}$ es el número de muestras ensayadas, aquí, igual a 20, y E el operador esperanza matemática. En esta matriz, posterior a su inversión, las varianzas quedan en la diagonal. Este método exige que la matriz de Fischer sea semidefinida positiva e invertible.

La bondad de ajuste se determinó mediante el test de hipótesis de chi - cuadrado, $\chi^{2}$. Para efectuar esta bondad de ajuste se clasificaron los datos experimentales en clases. El número de grados de libertad se determinó restando al número de clases el número de parámetros que, en este caso, fueron dos y, luego, restando uno. La evaluación se realizó considerando un nivel de confianza del $95 \%$. A partir de los datos experimentales, el valor del chi - cuadrado se determinó haciendo uso de:

$$
\chi^{2}=\sum_{i=1}^{K} \frac{\left(O_{i}-E_{i}\right)^{2}}{E_{i}}
$$

donde, $\mathrm{O}_{\mathrm{i}}$ es el valor observado de frecuencia en la clase $\mathrm{i}, \mathrm{E}_{\mathrm{i}}$ es el valor esperado de la frecuencia teórica en la clase i, k es el número de clases. Luego, el número de grados de libertad es igual a $\mathrm{k}-2-1$. El valor obtenido de la ecuación (7) se comparó con el valor de chi-cuadrado con un nivel de confianza de $95 \%$, a determinados números de grados de libertad.

En la tabla II se resumen los resultados numéricos obtenidos para cada una de las tres temperaturas de recocido empleadas en la aleación bajo estudio y los diferentes periodos de envejecimiento. Están los valores medios de la microdureza Vickers y su desviación estándar, obtenidos de cada uno de los seis conjuntos de 20 indentaciones, cada uno. También, se muestran los parámetros de Weibull estimados mediante el método de los mínimos cuadrados, a partir de los seis diagramas de Weibull y las dispersiones de los mismos obtenidas mediante el empleo de la matriz de información de Fischer. En la última columna, está el resultado del test de hipótesis. Desde 

KINETICS AND STATISTICAL ANALYSIS OF PRECIPITATION IN A CU-0.49Co-0.44TI ALLOY, BY MICROCALORIMETRY AND MICROHARDNESS MEASUREMENTS

una perspectiva puramente estadística, puede decirse que la estimación de los parámetros de Weibull y sus respectivas dispersiones caracterizan a la aleación bajo estudio, en la mayoría de los casos aquí considerados.

Se hace notar, en la tabla II, los elevados valores de las dispersiones de la microdureza Vickers, situación que también pudo observarse en los diagramas de Weibull. Este hecho es, precisamente, uno de los que permite hacer el análisis de los valores de microdureza Vickers empleando una función de distribución de probabilidad acumulativa. Hay que observar, además, que la mayoría de las muestras analizadas cumple con el test de bondad de ajuste de chi - cuadrado con un $95 \%$ de confiabilidad, es decir, los datos experimentales están regidos por funciones de distribución de probabilidad acumulativa de Weibull, con los parámetros allí indicados. En el único caso donde resultó rechazado el test de bondad de ajuste, si bien era posible hacer un ajuste con funciones de distribución de Weibull, el conjunto de datos requerían de la postulación de un tercer parámetro, el cual no fue estudiado aquí. Obsérvese, además, que para este caso existe sólo solución sólida sin presencia de fases precipitadas.

A partir del análisis de las trazas calorimétricas (Fig. 1) y del análisis estadístico de las mediciones de microdureza (Tabla II), podemos apreciar que, a medida que se incrementa el tiempo de envejecimiento, a igual temperatura de recocido, el valor del parámetro m se incrementa. De acuerdo con las curvas calorimétricas, a los $653 \mathrm{~K}, 0$ ks, se tiene sólo solución sólida, no hay presencia de precipitados, mientras que, a $723 \mathrm{~K}$, ya se ha iniciado la precipitación de la fase CoTi. Igual fenómeno ocurre a los 793 K. La presencia del precipitado modifica el tipo de función de distribución de Weibull, pasando de una de tres parámetros, sin formación de precipitado, a otra de dos parámetros, cuando está presente la fase precipitada. El aumento del parámetro m significa que la dispersión de los valores de microdureza disminuye. Nótese, en la tabla II, el significativo incremento del parámetro m a $723 \mathrm{~K}$, temperatura que coincide con el máximo valor medio de microdureza alcanzado, cuando se pasa de un tiempo de envejecimiento de 0,6 ks a 12,6 ks.

Conviene enfatizar que, por muy simple que sea el método de ajuste con mínimos cuadrados, al aplicarlo en una función de distribución de Weibull, de dos parámetros, como la ecuación (3), hay que tener cautela y considerarlo como una primera aproximación. En algunos casos, esta primera aproximación puede resultar una muy buena aproximación, pero hay que tener en consideración que pueden suceder hechos como el aquí registrado, es decir, la incorporación de un parámetro adicional.

\section{CONCLUSIONES}

Las observaciones anteriores permiten concluir lo siguiente:

- Mediante experiencias no isotérmicas se determinaron los parámetros entalpimétricos de la reacción de precipitación de CoTi. Utilizando el método de Kissinger modificado se obtuvo la energía de activación, E, de la reacción.

- Además, se determinaron los parámetros cinéticos, n y A, de la ecuación de Johnson-MehlAvrami para la precipitación de la fase CoTi. Los valores de $\mathrm{n}$, cercanos a la unidad, permiten indicar que el proceso de nucleación y crecimiento ocurre a partir de una solución sólida.

- Se observa un incremento de dureza máximo de la matriz, cuando el material es recocido a $723 \mathrm{~K}$ durante $12,6 \mathrm{ks}$, debido a la precipitación de la fase CoTi.

- Las mediciones de microdureza Vickers, a distintas temperaturas y distintos tiempos de envejecimiento, siguieron funciones de distribución de probabilidad acumulativa de Weibull de dos parámetros, para muestras con fase precipitada CoTi, con un nivel de confianza del 95 $\%$, determinado con el test de bondad de ajuste de chi-cuadrado. Excepto las muestras sin formación de precipitados, que siguieron una función de Weibull de tres parámetros.

- A medida que se incrementaba el tiempo de envejecimiento, para igual temperatura de recocido y observando las respectivas trazas calorimétricas, la aleación incrementaba el valor del parámetro m, de Weibull, es decir, la dispersión de los valores de microdureza disminuía. Lo anterior se atribuye a la precipitación de la fase CoTi.

\section{Agradecimientos}

Los autores desean agradecer al Fondo Nacional de Desarrollo Científico y Tecnológico (FONDECYT), Proyecto № 1090010, por el apoyo financiero, y al Departamento de Ciencia de los Materiales, Facultad de Ciencias Físicas y Matemáticas de la Universidad de Chile por las facilidades otorgadas para desarrollar este estudio.

\section{UNIDADES Y NOMENCLATURA}

$\begin{array}{ll}T & \text { Temperatura }(\mathrm{K}) \\ t & \text { Tiempo }(\mathrm{ks}) \\ F & \text { Función de probabilidad acumulativa }\end{array}$


H Variable aleatoria, microdureza Vickers

$\mathrm{H}_{0} \quad$ Parámetro de Weibull de escala

m Parámetro de Weibull, módulo de Weibull

p Número de orden

N Número de datos experimentales

F(HV) Probabilidad acumulativa de microdureza Vickers

HV Medidas experimentales de microdureza Vickers (HV)

$r_{i j} \quad$ Elemento de una matriz

$\theta_{i}, \theta_{j} \quad$ Variables de derivación

$\chi^{2} \quad$ Chi - cuadrado

$k \quad$ Número de clases

$\mathrm{O}_{i} \quad$ Valor observado

$E_{j}^{i} \quad$ Valor esperado

$\overrightarrow{H V} \quad$ Valor medio de microdureza Vickers

$\Delta \bar{H} v \quad$ Desviación estándar de microdureza Vickers

$\Delta m \quad$ Dispersión del módulo de Weibull

$\Delta H_{0} \quad$ Dispersión del parámetro de Weibull de escala

$\chi_{95 \%}^{2} \quad$ Chi - cuadrado con un $95 \%$ de confiabilidad

$\mathrm{H}_{L} \quad$ Parámetro de Weibull para probabilidad nula de microdureza Vickers

\section{REFERENCIAS}

[1] A. Varschavsky y E. Donoso, J. Termal Anal. Cal. 68 (2002) 231-241.

[2] E. Donoso, Rev. Metal. Madrid 37 (2002) 492 498.

[3] A. Varchavsky y E. Donoso, J. Termal Anal. Cal. 74 (2003) 41-56.

[4] L.Mineau, S. Hamar-Thibault y C.H. Allibert, Phys. Stat. Sol. (a) 134 (1992) 93-105.

[5] S. Nagarjuna, K.K. Sharma, I. Sudhakar y D.S. Sarma, Mater. Sci. Eng. A 313 (2001) 251-260.

[6] I.S. Batra, A. Laik, G.B. Kale, G.K. Dey y U.D. Kulkarni, Mater. Sci. Eng. A 402 (2005) 118 125.

[7] E. Donoso, G. Díaz y J.M. Criado, J. Thermal Anal. Cal. 91 (2008) 491-495.
[8] G. Fortina y M. Leoni, Metal. Italiana 10 (1972) 470-480.

[9] Z. Sierpinski y J. Gryziecki, Mater. Sci. Eng. A 264 (1999) 279-285.

[10] Z. Sierpinski y J. Gryziecki, Z. Metallkd. 89 (1998) 551-553.

[11] E. Donoso, M.J. Diánez, M.J. Sayagués, J.M. Criado, A. Varschavsky y G. Díaz, Rev. Metal. Madrid 43 (2007) 117- 124.

[12] H.U. Pfeifer, S. Bhan y K. Schubert, J. Lesscommon Metals 14 (1968) 291-302.

[13] J.L. Murray, Bull. Alloy Phase Diagrams 3 (1982) 74-78.

[14] K.P. Gupta, J. Phase Equilibria 24 (2003) $272-$ 275.

[15] W. Weibull, Ingenios Vetenskap Akad. Handl. 151 (1939) $1-45$.

[16] N.M. Pugno, J. Phys.: Condens. Mater. 18 (2006) S1.971-1.990.

[17] C. Lu, R. Danzar y F. Dieter Fisher, Phys. Rev. E 65 (2002) 067102: 1-4.

[18] M. Elgueta, G. Díaz, S. Zamorano y P. Kittl, J. Mater. Design 28 (2007) 2.496-2.499.

[19] J-M. Schneider, M. Bigerelle y A. Iost, Mater. Sci. Engng. A 262 (1999) 256-263.

[20] M.T. Lin, D.Y. Jiang, L. Li, Z. Lu, T.R. Lai y J.L. Shi, Mater. Sci. Eng. A 351 (2003) 9-14.

[21] Z. Tóth, A. Nagy, G. Steinbach y A. Juhász, Mater. Sci. Eng. A 542 (2004) 387-389.

[22] A.Varschavsky y E. Donoso, Mater. Lett. 57 (2003) 1266-1271.

[23] A. Varschavsky y E. Donoso, Mater. Sci. Eng. A 145 (1991) 95-107.

[24] E.J. Mittemeijer, L. Cheng., P.J. Van der Shaaf, C.M. Brakman y B.M. Korevaar, Metall. Trans. A 19 (1988) 925-932.

[25] A.M. Brown y M.F. Ashby, Acta Metall. 28 (1980) 1.085-1.101.

[26] T. Ozawa, J. Thermal Anal. 9 (1976) 369-373.

[27] J.W. Christian, The theory of transformation of metals and alloys. Part I: Equilibrium and general kinetics theory, Pergamon Press, EE. UU., 1975, p. 542. 